\title{
Fabrication of gold nanoparticles in Therminol VP-1 by laser ablation and fragmentation with fs pulses
}

\author{
R. Torres-Mendieta ${ }^{1}$, R. Mondragón ${ }^{2}$, E. Juliá ${ }^{2}$ O. \\ Mendoza-Yero ${ }^{1}$, E. Cordoncillo ${ }^{3}$, J. Lancis $^{1}$ and G. \\ Mínguez-Vega ${ }^{1}$ \\ ${ }^{1}$ GROC, UJI, Institut de Noves Tecnologies de la Imatge (INIT), Universitat Jaume \\ I., 12080, Castelló, Spain. \\ ${ }^{2}$ Departmento de Ingeniería Mecánica y Construcción, Universitat Jaume I., 12071, \\ Castelló, Spain. \\ ${ }^{3}$ Departmento de Química Inorgánica y Orgánica, ESTCE, Universitat Jaume I., \\ 12071, Castelló, Spain. \\ E-mail: mendieta@uji.es
}

\begin{abstract}
.
This paper reports a physical method to produce highly pure, size controlled and well dispersed spherical gold nanoparticles (NPs) in Therminol VP-1 by pulsed laser ablation in liquids (PLAL) using a $30 \mathrm{fs}$ Ti:Shappire laser at a fluence of $1 \mathrm{~J} / \mathrm{cm}^{2}$. A second photo-fragmentation of the ablated colloid solution by subsequent treatment with the same laser light yields a mean size and size dispersion of the NPs of $58 \pm 31 \mathrm{~nm}$. A study of the nanofluid properties reveals a low agglomeration over time and an enhancement of thermal conductivity of base fluid by up to $4 \%$. These results improve the characteristics of the current nanofluids in thermal oils that may have a potential impact in the improvement of the harvesting of solar light.
\end{abstract}




\section{Introduction}

Pulsed Laser Ablation in Liquids (PLAL) is a technique that has gained a lot of attention since 1987 when, for the first time, Patil et al. (1) reported the laser ablation of a solid iron target in water. Lately, it was also discovered that by means of this technique is possible the production of nanoparticles (NPs). In PLAL, the extraction mechanism is usually interpreted on the basis of different physical and chemical processes. The physical processes are governed by the laser parameters and the chemical processes mainly by the interaction between the particles and the base fluid. To enhance the characteristics of the NPs, after the ablation, the colloidal solution is irradiated again by the laser to promote another photo-fragmentation process of the NPs in the fluid $(2 ; 3)$. In particular, the strongest fragmentation effect in the second process is obtained when laser fluence is smaller than or about the breakdown threshold of the liquid. Hence, the selection of experimental parameters such as the light wavelength, laser fluence, ablation time, repetition rate, or the base fluid itself can modify the shape and size distribution of particles (4). For example, the ablation process with laser pulses of few femtoseconds temporal widths allows us to achieve higher purity in the final fluid as well as lower heat affected zone onto the sample than with nanosecond or picosecond pulses $(5 ; 6)$. Also, the laser-plume interaction is minimized, which makes the NPs generation process more controllable and the achievement of breakdown threshold in the fluid easier.

Applications of the PLAL technique and the photo-fragmentation additional process include, but are not limited to, the production of NPs of metallic and semiconductor materials (7-11), the morphological modification of nano-structures $(12 ; 13)$, or the synthesis of nanomaterials with chemical active fluids without needing a physical target $(14 ; 15)$. Synthesized like-flower NPs with potential abilities for surface enhanced Raman Scattering was also proved by Lu et al. (16). Recently, the selection of the base fluid has been shown to be a key parameter in many applications such as the development of high speed and frequency electronic devices $(17 ; 18)$ or the synthesis of non-toxic NPs that present infrared emission for biomedical applications $(19 ; 20)$.

On the other hand, the concept of adding small solid particles into a fluid to increase the thermal properties of the suspension has been practiced since 1873 (21). However, most of the studies were performed using suspensions of millimeter- or micrometer- sized particles, which led to problems such as poor suspension stability and channel clogging, which limits its practical applicability. To solve these drawbacks in 1995, S. U. S. Choi proposed the use of nanofluids to increase the thermal conductivity of heat transfer fluids (22). Nanofluids are defined as dilute suspensions with solid NPs.

Thermal oils are widely used in several industrial applications such as solar thermal power plants. Solar energy is one of the best sources of renewable energy with minimal environmental impact. Nowadays, the improvement of the harvesting of solar light is a challenge. One way to increase the efficiency in solar thermal collectors is by fabricating nanofluids using a thermal oil such as Therminol VP-1 as a base fluid $(23 ; 24)$.

In this context, Wang et al. (24) experimentally demonstrated that the fabrication 
of spherical gold NPs with sizes around $121 \mathrm{~nm}$ and concentrations of $0.05 \%$ (mass percentage) in Therminol VP-1 can enhance its thermal conductivity by up to $6.5 \%$, increasing in this way the harvesting of energy in solar plants. This happens at least 3 days before that the agglomeration of NPs and increment of viscosity appears (it is considered that an increment in nanofluid viscosity is a drawback due to the associated increment in fluid pumping costs). Note that, in the procedure followed by Wang et al., gold NPs are commonly produced in form of colloidal suspensions into a given solution separately, and subsequently dispersed into Therminol VP-1. Hence, the agglomeration of NPs cannot be disregarded, leading to poor suspension stability and high shear viscosity. In order to mitigate this unwanted effect alternative procedures based on the production of NPs directly into the fluids have been proposed (25-28). Unfortunately, most of them (i.e., wet techniques) demonstrate low effectiveness to avoid the NPs agglomeration and/or show pollution problems due to the surfactants used in the synthesis of NPs.

In this paper we experimentally demonstrated the fabrication of a thermal oil nanofluid based on femtosecond laser ablation and re-fragmentation of bulk gold inside Therminol VP-1. The morphology, purity, mean size, size dispersion of our NPs and the stability and thermal conductivity enhancement of the nanofluid are determined. Owing to the fact that no chemical stabilizer, reductor or dispersant are employed to synthesize the NPs and thanks to the photo-fragmentation process we obtain small particle size and the agglomeration over time is significantly alleviated. This may have a potential impact in using this nanofluid in real applications as in thermal power plants.

\section{Experimental setup}

In our experiment we produce gold nanoparticles in Therminol VP-1 by PLAL followed by a second photo-fragmentation process. The first stage of the experiment involves a laser ablation process where the ejected fragments of material produced by the ablation are captured in a liquid (in this case we used Therminol VP-1). It was carried out with a Ti:Sapphire laser (Femtopower Compact Pro, Femtolasers), that emits pulses of $30 \mathrm{fs}$ intensity full width at half maximum (FWHM) with a central wavelength of $800 \mathrm{~nm}$, maximum energy per pulses of $0.5 \mathrm{~mJ}$, and $1 \mathrm{kHz}$ repetition rate. Before exit the ultrashort pulses pass through a user-adjustable post-compression stage based on two fused silica Brewster prisms which allows us to control the dispersion in the beam delivery path. The energy of the pulses was measured with an analogical power-meter (Spectra Physics, Model 407-A) and controlled by using a set of calibrated neutral density filters. The cross-section of the pulsed beam was slightly elliptical, with a nominal beam diameter of $15 \mathrm{~mm}$ in accordance with the $1 / e^{2}$ criterium, and a beam propagation factor lower than two. To reduce optical aberrations and increase the spatial uniformity, the beam passes through a $2 x$ all-mirror beam expander. In addition, an iris of $6 \mathrm{~mm}$ of diameter is placed before the focusing optics.

A gold disc of thickness $1 \mathrm{~mm}$ and diameter $6.5 \mathrm{~mm}$ (99.99\% purity) was used as the 
target. The gold target was placed at the bottom of a glass vessel (cuvette) filled with Therminol VP-1. The thickness of the fluid layer above the target was about $7 \mathrm{~mm}$. The cuvette was attached to a 2-D motion controlled stage which can be moved at a constant speed during the experiment. The target was irradiated from the air-liquid interface by means of a focused beam with a lens of $75 \mathrm{~mm}$ focal length onto the target surface while moving the target, perpendicular to the beam propagation axis, at a constant velocity of $4.5 \mathrm{~mm} / \mathrm{s}$. The dispersion introduced by the liquid and the lens was compensated with the help of the above mentioned post-compression stage. To adjust the duration of the ablation process, an electronically controlled shutter was utilized. In these conditions, after $15-17 \mathrm{~min}$ we obtained a brown coloration in the aqueous solution due to the presence of the gold nanoparticles in the fluid.

In the following experimental stage, we filled a $10 \mathrm{ml}$ glass cuvette with the NP colloidal solution obtained from the first stage of the experiment. Then, the cuvette was irradiated by using the light from the Ti:Sapphire laser, previously focused onto its center with the same lens, while the suspension was stirred by a magnet to homogenize the photo-ablation process which consist of a fragmentation of the biggest particles into smaller particles by means of a second ablation process.

Under free propagation, the fluence of the laser beam at the focal point of the lens is about $1 \mathrm{~J} / \mathrm{cm}^{2}$. However, due to the refraction effects this value decreases until $0.6 \mathrm{~J} / \mathrm{cm}^{2}$ approximately. Please also note that the laser fluence inside the cuvette is not constant, but decreasing as we move away from the focal position towards the lens.

\section{Results and discussion}

In this section by using Transmission Electronic Microscopy (TEM), Energy Dispersive X-ray Spectroscopy (EDX), High Resolution Transmission Electronic Microscopy (HRTEM) and Dynamic Light Scattering (DLS) we determine the morphology, purity, mean size, size dispersion, and stability of the fabricated and commercial NPs. This section is divided in two subsections, the production of NPs where we will focus in the properties of the nanofluid just after its fabrication and the section called nanofluid properties where we will study some characteristics of our nanofluid such as the evolution of the mean size of the particles through the time and the nanofluid thermal conductivity.

\subsection{Production of nanoparticles}

To study the quality of the NPs fabricated with the $f s$ laser we compare their properties with commercial NPs added to Therminol VP-1. In order to obtain highly dispersed NPs, similar to the ones obtained by the PLAL technique, commercial gold NPs are acquired in aqueous suspension from Sigma Aldrich. NPs are stabilized in $0.1 \mathrm{mM}$ of phosphate buffered saline (PBS) solution and the primary particle diameter is $50 \mathrm{~nm}$. To prepare Therminol VP-1/gold nanofluids, first water content is removed from the aqueous nanofluid by evaporation in a hot plate. The solid product obtained is then 
dispersed in Therminol VP-1 with the help of an ultrasound probe and a concentrated nanofluids is obtained. The sonication time varies from 5 to $10 \mathrm{~min}$ depending on the sample volume. Finally, nanofluids are diluted with pure Therminol VP-1 to achieve the same optical density and solid content in all the cases. Optical density has been calculated by measuring the radiation absorption of a laser beam at $635 \mathrm{~nm}$ (Model CPS180, ThorLabs) with a photodiode (Model SM05PD1B, Thorlabs) in a rectangular quartz cuvette.

To determine the morphology of the gold NPs and the purity of the final material, a droplet of the colloidal suspension of Therminol VP-1 with NPs was dispersed onto a carbon-coated copper-based TEM grid. The liquid content was then removed with the help of an absorbent paper so the solid particles can remain in the grid surface. This technique allows obtaining dry particles highly dispersed and the morphology and particle size of the primary particles can be determined. The micrographs shown in Figure 1 a) - c) were obtained with a TEM (JEOL 2100 microscope) operating at a voltage of $100 \mathrm{kV}$. Figure $1 \mathrm{a}$ ) and Figure $1 \mathrm{~b}$ ) correspond to samples synthesized by PLAL and PLAL followed by the second photo-fragmentation process, respectively, whereas in Figure $1 \mathrm{c}$ ) the sample was generated by addition of commercial gold NPs into Therminol VP-1 as explained above. From the results, it is clear that in contrast with the quasi-ellipsoidal particles shown in Figure $1 \mathrm{c}$ ), the morphology of the particles synthesized with PLAL is mostly spherical.
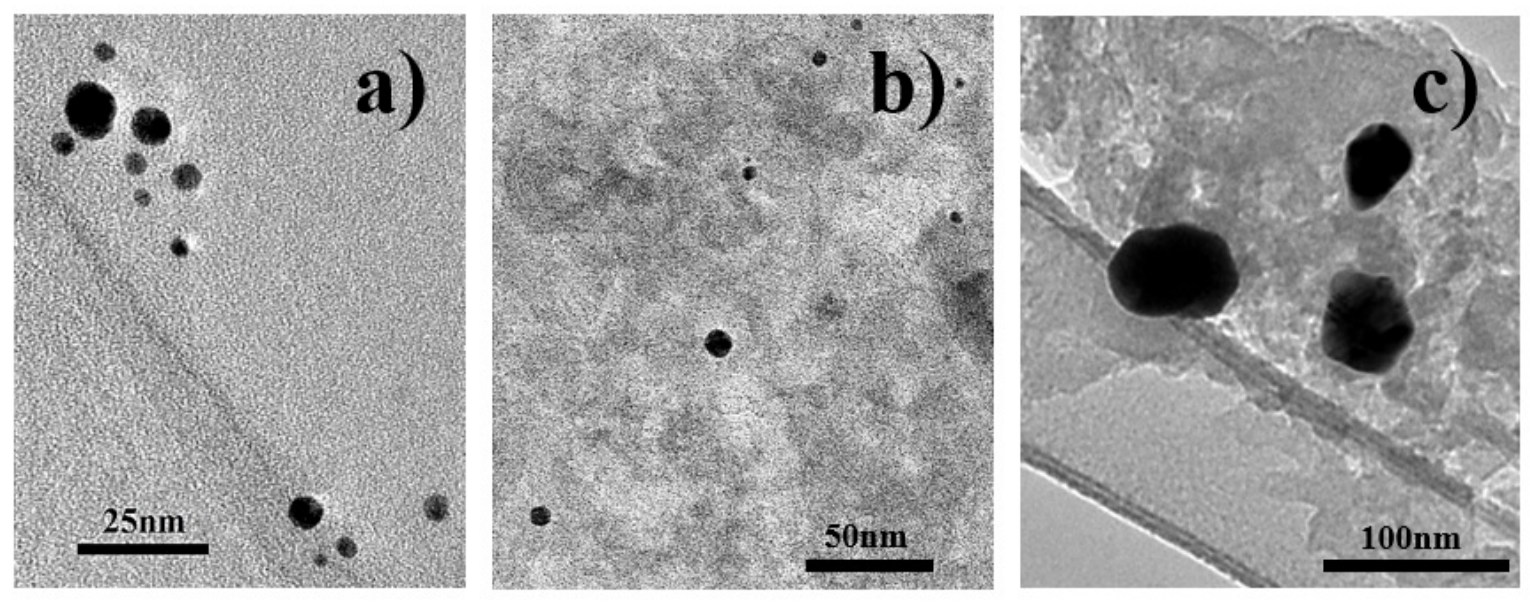

Figure 1. TEM micrographs of samples fabricated by a) PLAL b) PLAL followed by the second photo-fragmentation process, and c) commercial NPs.

To ensure the purity of the gold NPs obtained with the femtosecond laser, an elemental composition of the NPs was determined using an EDX system attached to the TEM (an Oxford Instruments INCA Penta FETX3). The EDX detected gold ( $\mathrm{Au}$ ), copper $(\mathrm{Cu})$ and carbon $(\mathrm{C})$ (see Figure $2 \mathrm{a}$ )). However, the presence of copper and carbon is caused by the fact that the grid substrate is made of copper and it contains a carbon film. In this direction, we also performed HRTEM measurements to see the crystallographyc planes of the gold NPs and corroborate our results. Figure 2 
b) shows four typical spherical Au particles with diameters less than $5 \mathrm{~nm}$, embedded in the thermal fluid. The clear lattice fringes indicate the high crystallinity. The inset in Figure 2 b) allows us to see the crystallographic planes. As might be expected, the $d$ interplanar spacings represented by $(h k l)$ Miller index obtained from HRTEM micrographs in Table 1 are similar to those included in Joint Committee on Powder Diffraction Standards (JCPDS) files for cubic Au crystals (JCPDS: 4-784). As one could extrapolate from these results, the syntethized particles of $\mathrm{Au}$ seem to be free from vacancies or defects in the crystal due to the mixture between gold and another element that comes from the fluid.
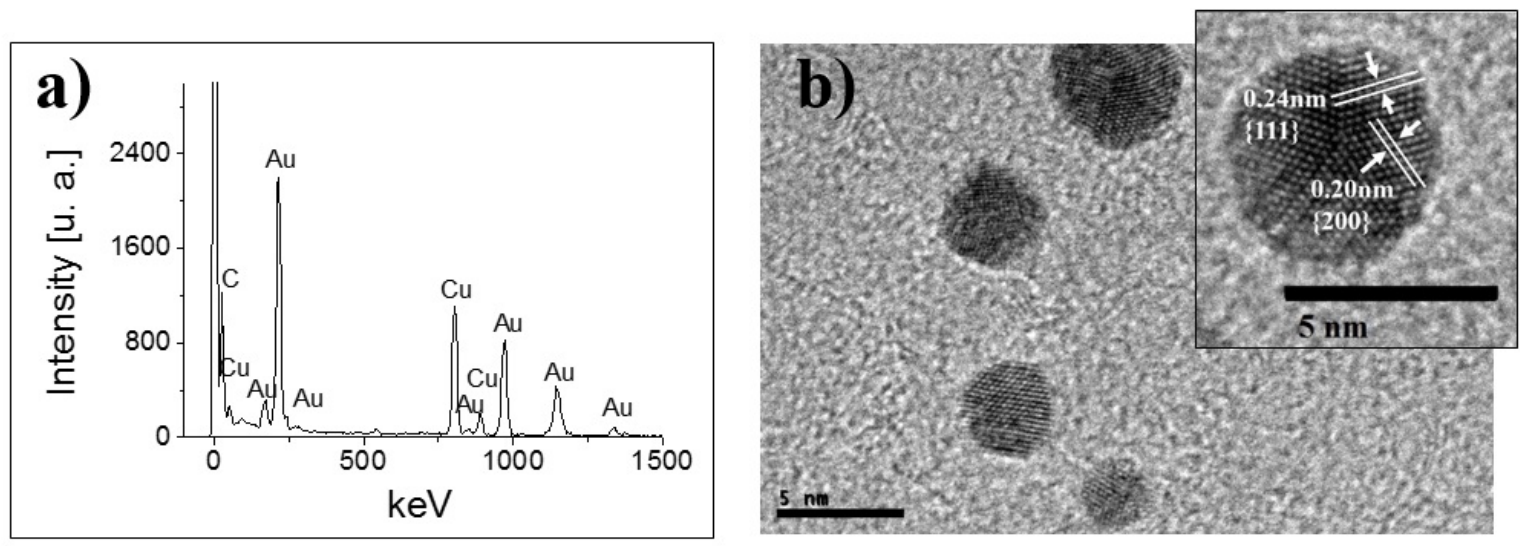

Figure 2. a) EDX spectrum of produced nanoparticles (the presence of carbon and copper is caused by the grid use to deposit the Au NPs) b) HRTEM micrography of produced nanoparticles.

Table 1. $d$-Spacing of the crystalline gold NPs measured from HRTEM micrographs.

\begin{tabular}{cccc}
\hline $\begin{array}{c}d \text {-Spacing } / \AA \\
\text { (experimental results) }\end{array}$ & $\begin{array}{c}d \text {-Spacing/ } / \AA \\
\text { (JPCDS: } 4 \text {-784 data) }\end{array}$ & $(h k l)$ values & Phase assignment \\
\hline 2.40 & 2.35 & $(111)$ & cubic \\
2.00 & 2.04 & $(200)$ & cubic \\
\hline
\end{tabular}

At this point, we determined the size distributions of the NPs by DLS just few minutes after the fabrication of the nanofluid. This technique provides the size distribution of the particles or agglomerates of particles as they are present in the nanolfuid in static conditions. To this end, we use a Zetasizer nano ZS (Malvern Instruments Ltd., UK) with a $173^{\circ}$ scattering angle. Particle size was assessed from Brownian motion by means of the Einstein-Stokes equation. Results are shown in Figure 3. Nevertheless, we have to take into account that the mean size and size distribution value depicted in Figure 3 is a rough value estimation of the original size of fabricated NPs because during the time spent between the measurement and the nanofluid fabrication the NPs could start to agglomerate and, in fact, the measured NPs 
size represents the size of agglomerates, but for our purposes (particle aglommeration dynamics study) this value can represent a good figure of merit.

From statistical size analysis, the mean size and size dispersion for the commercial NPs were $255 \pm 192 \mathrm{~nm}$ at FWHM. In contrast, the nanofluid produced with PLAL technique shows a mean size of $122 \mathrm{~nm}$, while the size dispersion was of $155 \mathrm{~nm}$. Furthermore, the nanofluid produced with the PLAL and the second photofragmentation process shows a mean size of $58 \mathrm{~nm}$ and the lower size dispersion (about $31 \mathrm{~nm}$ of FWHM). These results show that the production of gold NPs by PLAL with the second photo-fragmentation process allows us decreasing the size dispersion and the mean size of the NPs inside the thermal oil, avoiding the agglomeration phenomenon in the production process. Consequently, by controlling the time of the second photofragmentation process, we are able to control the characteristics of the NPs in the nanofluid.

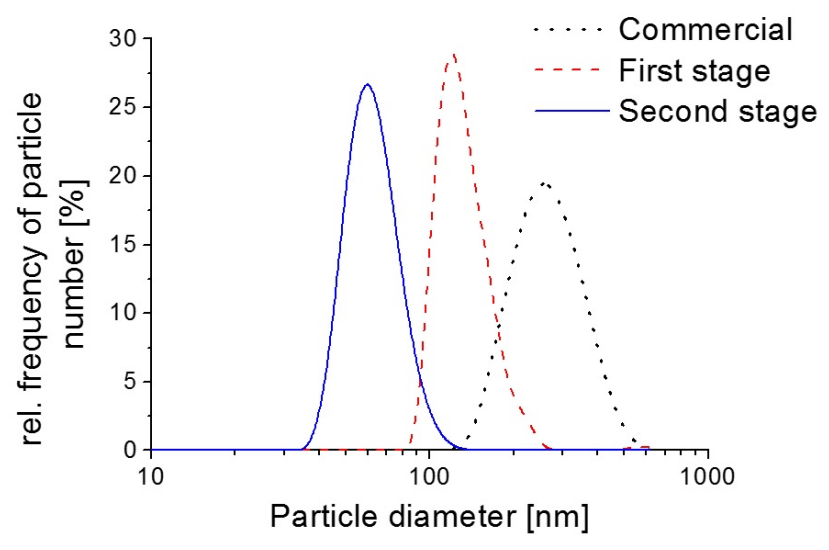

Figure 3. Comparison between distribution of sizes obtained for each fluid.

\subsection{Nanofluid Properties}

One of the most important characteristics of nanofluids is that they can remain for very prolonged periods of time without significant settling or loss of stability due to the Brownian motion. As the particle size decreases, Brownian motion becomes predominant over the Stokes sedimentation $(29 ; 30)$. Stability in colloid science is used not only in the thermodynamic sense but also in a strictly colloidal sense. Colloidally stable means that the colloidal particles do not settle and do not agglomerate at a significant rate. The classical DLVO (Derjaguin- Landau-Verwey-Overbeek) theory predicts the stability of the nanofluids by knowing the total energy of interaction between two particles (31). In the range of sizes of nanofluids, the ratio particle surface to particle volume is so high that all the interactions are controlled by short-range forces like Van der Waals attraction force. As a consequence of the attractive nature of the forces presented, when primary particles are suspended in a fluid they have a tendency to agglomerate when come into contact due to their Brownian motion. These agglomerates remain 
in suspension providing a stable nanofluid unless the agglomerate size becomes bigger enough to settle.

The stability of Au/Therminol VP-1 nanofluid was determined by the analysis of mean particle size of Au nanoparticles at different times. To do that, the mean size of the NPs inside the fluid was measured 12 times in 96 hours. The results are shown in Figure 4. The average size of Au particles increases gradually within the first 6 hours due to the clusters formation. After that time, agglomerate size keeps constant and nanofluids can be considered stable for at least 96 hours. From Figure 4 one can see that in comparison with the use of commercial NPs, our nanofluid made in two experimental stages reaches its stability with clusters size of $523 \mathrm{~nm}$, while the nanofluid synthesized with commercial NPs does with $968 \mathrm{~nm}$. So the nanofluid generated by PLAL with the second photo-fragmentation process alleviates in great measure the unwanted agglomeration phenomena.

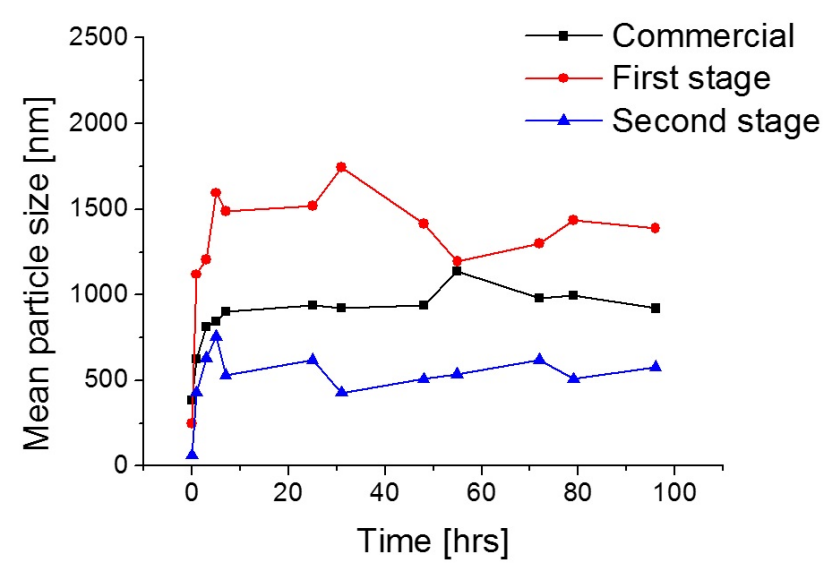

Figure 4. Comparison between agglomeration dynamics trough the time.

As mentioned above, nanofluids are used as heat transfer fluids due to their improved thermal properties. The addition of solid particles with higher thermal conductivity than the base fluid results in a thermal conductivity enhancement that can be predicted by the Maxwell equation. In this work the thermal conductivity of the nanofluids produced by PLAL (after the first and second experimental stages) and the commercial nanofluid was measured at $80^{\circ} \mathrm{C}$ by means of the transient hot wire technique, using a KD2 Pro conductimeter (Decagon Devices Inc.). Six measurements were done for each nanofluid sample so the experimental error could be determined at a $95 \%$ of confidence level. Results are shown in Table 2. It can be observed that the thermal conductivity increases when nanoparticles are suspended in the base fluid. The enhancement achieved depends on the size and shape of the particles and clusters of particles in the nanofluid, acording to reference (32). The best results were obtained for the second experimental stage sample in which particles present the lowest size, while the first experimental stage sample with the biggest agglomerates presents the lowest thermal conductivity enhancement. 
Table 2. Thermal conductivity enhancement at $80^{\circ} \mathrm{C}$.

\begin{tabular}{ccc}
\hline Sample & $k(W / m K)$ & $\Delta k(\%)$ \\
\hline Therminol VP-1 & $0.1280 \pm 0.0030$ & - \\
Commercial & $0.1320 \pm 0.0018$ & 3.13 \\
First stage & $0.1282 \pm 0.0015$ & 0.13 \\
Second stage & $0.1332 \pm 0.0014$ & 4.06 \\
\hline
\end{tabular}

\section{Conclusions}

In this paper, we have successfully produced highly pure, size controlled and well dispersed gold NPs in Therminol VP-1 by PLAL and a second photo-fragmentation process without using any chemical additives. The analysis of TEM and HRTEM micrographs along with EDX spectra exhibit that our NPs have spherical shape, the nanofluids show no pollution and the crystallographyc planes suggest that there are no defects due to impurities. We also observed that thanks to the photo-fragmentation process, in general we obtain smaller NPs than $58 \mathrm{~nm}$ of mean size. This small NPs size is difficult to be obtained with other methods due to the fact that NPs tend to agglomerate very fast in Therminol VP-1. Moreover a study of the stability over time reveals that the NPs produced by ablation and the following fragmentation process with laser acquires a size that is at least $54 \%$ lower than in the rest of the cases. Finally, thermal conductivity measurements revealed that nanofluids present higher thermal conductivity than the based fluid making them suitable for their use as heat transfer fluids. The nanofluid produced by ablation and the following fragmentation process of NPs with femtosecond laser light showed the highest thermal conductivity enhancement. All these results represent a great advantage over other methods to produce NPs in thermal oil that may have a potential great impact in the improvement of the harvesting of solar light with nanofluids.

\section{Acknowledgements}

This work was funded by the Generalitat Valenciana through the programme $(P R O M E T E O \backslash 2012 \backslash 021)$, and by University Jaume I through the project $P 1 \cdot 1 B 2013$ - 53. The authors are also very grateful to the SCIC of the Universitat Jaume I for the use of the femtosecond laser. Finally, R. Torres-Mendieta greatfully acknowleadges the Generalitat Valenciana support from the Santiago Grisolia scholarship GRISOLIA/2013/015.

\section{References}

[1] P. P. Patil, D. M. Phase, S. A. Kulkarni, S. V. Ghaisas, S. K. Kulkarni, S. M. Kanetkar, S. B. Ogale and V. G. Bhide 1987 Phys. Rev. Lett. 58238 
[2] F. A. Videla, G. A. Torchia, D. C. Schinca, A. B. Scaffardi and P. Moreno $2010 J$. Appl. Phys. 107114308

[3] S. Besner, A. V. Kabashin and M. Meunier 2007 Appl. Phys. A 88269

[4] A. Pyatenko, et al. 2013 Laser Photonics Rev. 7596

[5] A. V. Kabashin and M. Meunier 2003 J. Appl. Phys. 947941

[6] A. H. Chin, R. W. Schoenlein and T. E. Glover 1999 Phys. Rev. Lett. 83336

[7] A. Menéndez-Manjón, P. Wagener and S. Barcikowski 2011 J. Phys. Chem. C 115 5108

[8] D. M. Popovic, J. S. Chai, A. A. Zekic, M. Trtica, M. Momcilovic and S. Maletic 2013 Laser Phys. Lett. 10026001

[9] H. S. Desarkar, P. Kumbhakar and A. K. Mitra 2013 Laser Phys. Lett. 10055903

[10] F. Hajiesmaeilbaigi, A. Mohammadalipour, J. Sabbaghzadeh, S. Hoseinkhani, and H. R. Fallah 2006 Laser Phys. Lett. 3252

[11] A. Hahn, S. Barcikowski and B. N. Chichkov 2008 JLMN 373

[12] S. Link, C. Burda, B. Nikoobakht and M. A. El-Sayed 2000 J. Phys. Chem. B 104 6152

[13] S. Link and M. A. El-Sayed 1999 J. Phys. Chem. B 1038410

[14] P. H. D. Ferreira, M. G. Vivas, L. De Boni, Jr. D. dosSantos, D. T. Balogh, L. Misoguti and C. R. Mendonca 2008 Opt. Express 20136

[15] B. Tangeysh, K. M. Tibbetts, J. H. Odhner, B. B. Wayland and R. J. Levis 2013 J. Phys. Chem. C 11718719

[16] W. Lu, M. Zheng, W. Chen, Z. Zhao and X. Duan 2012 Phys. Chem. Chem. Phys. 1411930

[17] Y. Kwon, et al. 2006 Electrochem. Solid-State Lett. 9 A34

[18] G. Wang, Y. Siu-Tung, M. Kevin and H. N. Munir 2008 Opt.Commun. 2811765

[19] G. K. Podagatlapalli, S. Hamad, S. P. Tewari, S. Sreedhar, M. D. Prasad and S. V. Rao 2013 J. Appl. Phys. 113073106

[20] C. J. Lin, at al. 2009 ACS Nano 3395

[21] J. C. Maxwell 1873 A Treatise on Electricity and Magnetism (UK:Clarendon Press)

[22] S. U. S. Choi 1995 In Proceedings of the 1995 ASME International Mechanical Engineering Congress and Exposition (USA) 12

[23] A. Lenert and E. N. Wang 2012 Sol. Energy 86253

[24] C. Wang, J. Yang and Y. Ding 2013 Progress in Natural Science: Materials International 23338

[25] J. Sylvestre, A. V. Kabashin, E. Sacher, M. Meunier and J. H. T. Luong 2004 JACS 1267176

[26] S. Cingarapu, D. Singh, E. V. Timofeeva and M. R. Moravek 2013 Int. J. Energy Res. 3851 
[27] M. L. Marin, K. L. McGilvray and J. C. Scaiano 2008 JACS 13016572

[28] S. Barcikowski and G. Compagnini 2013 Phys. Chem. Chem. Phys. 153022

[29] D. J. Shaw 1991 Introduction to colloid and surface chemistry (UK:ButterworthHeinemann)

[30] H. E. Bergna and W. O. Roberts 2006 Colloidal silica: Fundamentals and applications (USA:CRC Taylor and Francis)

[31] D. Quemada and C. Berli 2002 Adv. Colloid Interface Sci. 9851

[32] J. W. Gao, R. T. Zheng, H. Ohtani, D. S. Zhu and G. Chen 2009 Nano Let. 94128. 